\title{
Carbamothioates in the synthesis of diaroyl sulfides; selectivity in the addition of diaroyl sulfides to acetylenic and ethylenic $\pi$-deficient compounds
}

\author{
Ashraf A. Aly, a $^{*}$ Alan B. Brown,

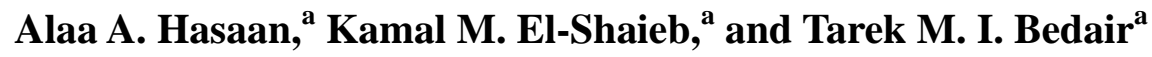 \\ ${ }^{a}$ Department of Chemistry, Faculty of Science, El-Minia University, 61519-El-Minia, Egypt \\ ${ }^{b}$ Chemistry Department, Florida Institute of Technology, 150 W University Blvd, Melbourne, \\ Florida 32901, U.S.A. \\ E-mail: ashrafaly63@yahoo.com
}

\begin{abstract}
Diaroyl sulfides were prepared in one-pot reaction from ethylenediamine and carbon disulfide, via reaction of in situ generated ethylene-bis-carbamodithioates with aroyl chlorides. The reaction of diaroyl sulfides with acetylenic and ethylenic $\pi$-deficient compounds such as dimethyl acetylenedicarboxylate, ethyl propiolate, (E)-1,4-diphenylbut-2-ene-1,4-dione and 1,4diphenylbut-2-yne-1,4-dione afforded the corresponding thionylated products. NMR spectroscopic data of the isolated products were discussed.
\end{abstract}

Keywords: Carbamodithioates, diaroyl sulfides, ethylenic and acetylenic $\pi$-deficient compounds, dithiols, aroylthionylation and NMR investigation

\section{Introduction}

Diaroyl sulfides were previously described in the literature ${ }^{1}$ and their synthesis remains of interest due to their biological importance. ${ }^{2}$ To the best of our knowledge there are no reported reactions of diacyl sulfides with $\pi$-deficient compounds, where as several 1-propyn-3-ols with excess acetic anhydride gave 1,1-diacetoxy-2-propenes. ${ }^{3}$

Reaction of thiolacetic acid with propiolaldehyde gave $\beta$-(acetylthio)acrolein. ${ }^{4}$ Reaction of thiolacetic acid with methyl propiolate gave methyl 3-(acetylthio)acrylate and a smaller amount of a double conjugate adduct. ${ }^{5}$

Interestingly, it was reported that the reaction of acetylenedicarboxylic acid with thiolacetic acid gave racemic and meso-2,3-bis(acetylthio)succinic acid. ${ }^{6}$ Haugwitz has reported that methanethioic-S-acid reacted with acrylaldehyde to afford the conjugate addition product. ${ }^{7}$ 
Aly et al. have investigated the reactions of thiols and thioamides with $\pi$-deficient compounds, ${ }^{8}$ and the antitumor and antioxidant activities of the resulting products. ${ }^{9}$ Herein we report the reactions of diaroyl sulfides and carbamothioates with various acetylenic and ethylenic $\pi$ deficient compounds.

\section{Results and Discussion}

The bis(dithiocarbamate) salt $\mathbf{1}^{10}$ was synthesized by adding carbon disulfide to a solution of ethylenediamine and triethylamine as outlined in Scheme 1. Also it was reported that the reaction of polymethylene diamines with aroyl chloride and ammonium thiocyanate under solid-liquid phase-transfer catalysis by polyethylene glycol-400 (PEG-400) yielded polymethylene-bis-aroyl thioureas in good-to-excellent yields. ${ }^{11}$

Under these conditions, salt 1 reacted with excess amounts of aroyl chlorides in the presence of a PEG 400-water mixture at $0{ }^{\circ} \mathrm{C}$, but instead of diaroyl ethylenebis-(carbamodithioates) 3a-c, the corresponding diaroyl sulfides $2 \mathbf{2 a}^{1 \mathrm{a}} \mathbf{c}^{\mathrm{a}}$ were obtained in good yields (Scheme 1). This one-pot reaction was synthetically useful.

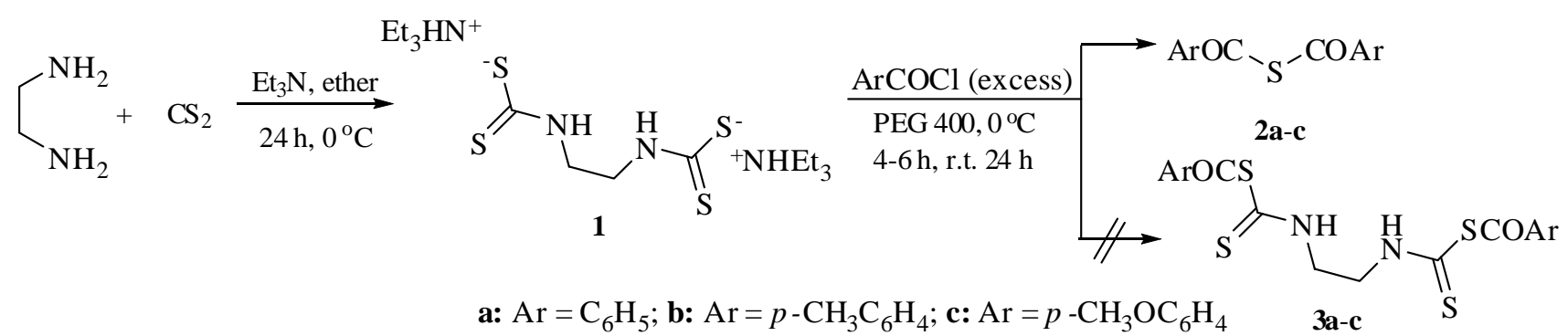

Scheme 1. Synthesis of diaroyl sulfides 2a-c. 2a: 90\%; 2b: 94\%; 2c: 96\%.

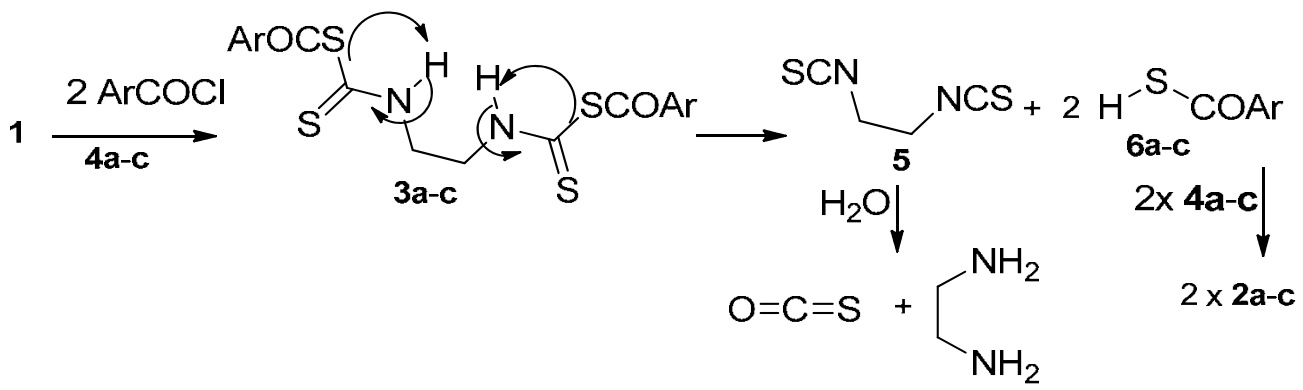

Scheme 2. Plausible mechanism for formation of diaroyl sulfides 2a-c

We attribute the conversion of salt $\mathbf{1}$ into diaroyl sulfides 2a-c to aroylation of $\mathbf{1}$ with aroyl chlorides 4a-c leading to 3a-c (Scheme 2). The diacyl ethylenebis(dithiocarbamates) 3a-c would 
then decompose, to give ethylenebis(isothiocyanate) $\mathbf{5}$ and two equivalents of thiolaroates 6a-c. (The reaction of primary amines with $\mathrm{CS}_{2}$ and acyl halides has been used as a synthesis of isothiocyanates ${ }^{12}$ ). Compounds $\mathbf{6 a - c}$ would undergo further aroylation to form compounds $\mathbf{2 a - c}$. Isothiocyanate $\mathbf{5}$ is proposed to undergo hydrolysis to ethylenediamine, with extrusion of carbon oxysulfide.

Reaction of two equivalents of diaroyl sulfides $2 \mathbf{2 a - c ^ { 1 a }}$ with one equivalent of dimethyl acetylenedicarboxylate (7) gave mainly 2,3-dimethyl bis(aroylthio)succinates 8a-c in good yields (Scheme 3). Mass spectrometry and elemental analyses of 8a-c proved the assigned molecular formulae. The IR spectra of 8a-c show no $\mathrm{C}=\mathrm{S}$ or $\mathrm{NH}$ groups. Proton and ${ }^{13} \mathrm{C}$ NMR spectra were consistent with the structures, and in particular the ${ }^{13} \mathrm{C}$ data showed the twofold symmetry. Connectivities were established using ${ }^{1} \mathrm{H}-{ }^{1} \mathrm{H}$ COSY, HMQC, and HMBC studies. It is worthy of note that the yields of the obtained products increase in the presence of electron donating groups on the aromatic moiety such as methyl and methoxy in $\mathbf{2 b}$ and $\mathbf{2 c}$. Evidently the reaction between two equivalents of $\mathbf{2 a - c}$ and $\mathbf{7}$ involves nucleophilic attack of $\mathbf{2 a - c}$ on the acetylenic $\pi$ bond in 7 (Scheme 3).

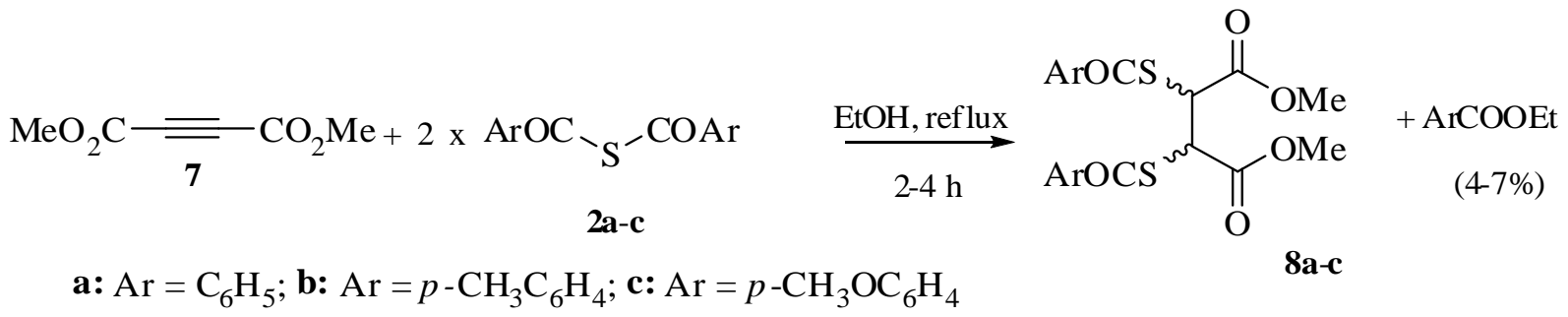

Scheme 3. Aroylthionylation of 7 by 2a-c. 8a: 4 h, 60\%; 8b: 2.5 h, 65\%; 8c: 2 h, $70 \%$.

We propose that the formation of adducts 8a-c begins with addition of the sulfur electron-pair of 2a-c to the triple bond of 7 to form salt $\mathbf{9}$ (Scheme 4). The carbanion formed by this nucleophilic attack can abstract a proton from the solvent to give intermediate $\mathbf{1 0}$ in addition to ethyl aryloate. Ethanol presumably removes an aroyl group from $\mathbf{9}$ in the form of ethyl aryloate as opposed to a free aroyl cation. Subsequently another molecule of $\mathbf{2}$ would add to $\mathbf{1 0}$ to form the stable adducts 8a-c together with a second molecule of ethyl aryloate (Scheme 4). This mechanism was supported by isolation of ethyl aryloates. All these reactions increase in rate with electron donation to the aroyl group; this observation supports a polar mechanism. 


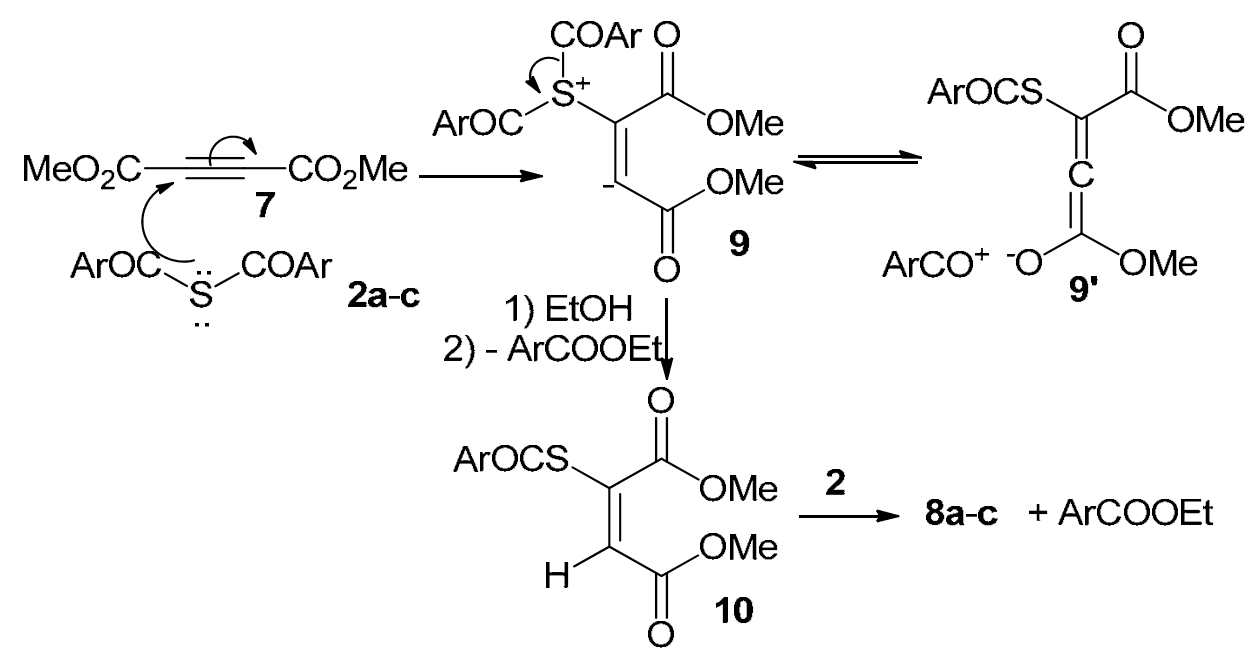

Scheme 4. Suggested mechanism of the reaction between $2 \mathbf{a}-\mathbf{c}$ and 7.

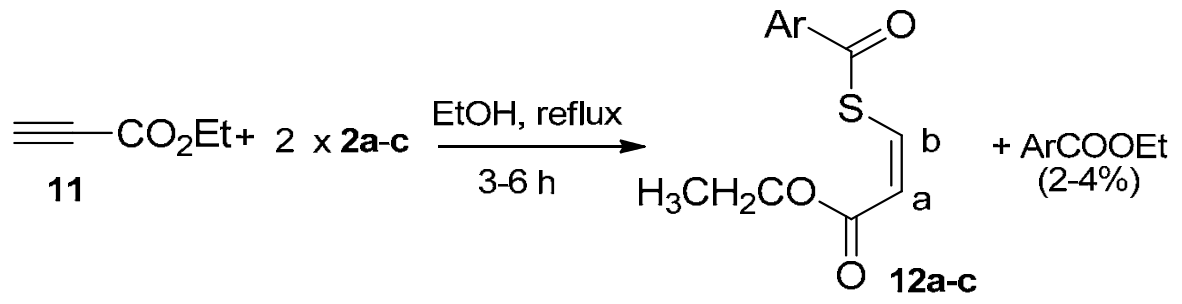

$\mathbf{a}: \mathrm{Ar}=\mathrm{C}_{6} \mathrm{H}_{5} ; \mathbf{b}: \mathrm{Ar}=p-\mathrm{CH}_{3} \mathrm{C}_{6} \mathrm{H}_{4} ; \mathbf{c}: \mathrm{Ar}=p-\mathrm{CH}_{3} \mathrm{OC}_{6} \mathrm{H}_{4}$

Scheme 5. Reaction of 2a-c with ethyl propiolate 11. 12a: 6 h, 72\%; 12b: 5 h, 76\%; 12c: 3 h, $82 \%$.

To further examine the generality of the reaction, we then studied the reactions of 2a-c with ethyl propiolate 11. In refluxing ethanol, the reaction afforded ethyl (2Z)-3(benzoylthio)acrylates 12a-c (Scheme 5). Although the reaction was begun by combining two equivalents of 2a-c with one equivalent of 11, the reactions reached completion when equal equivalents reacted of both 2a-c and 11. Again, mass spectrometry and microanalysis proved the formulae of 12a-c. The structures of 12a-c follow from IR, ${ }^{1} \mathrm{H} N M R$, and ${ }^{13} \mathrm{C} N M R$ spectroscopy; connectivities were established using ${ }^{1} \mathrm{H}-{ }^{1} \mathrm{H}$ COSY, HMQC, and HMBC studies. The vinylic protons appeared as two doublets with $J=10.0-10.3 \mathrm{~Hz}$, demonstrating the cisgeometry of the ethylenic bond. A vinylic coupling constant of $10 \mathrm{~Hz}$ is unusually small for a trans coupling but normal for a cis coupling. ${ }^{13}$ 


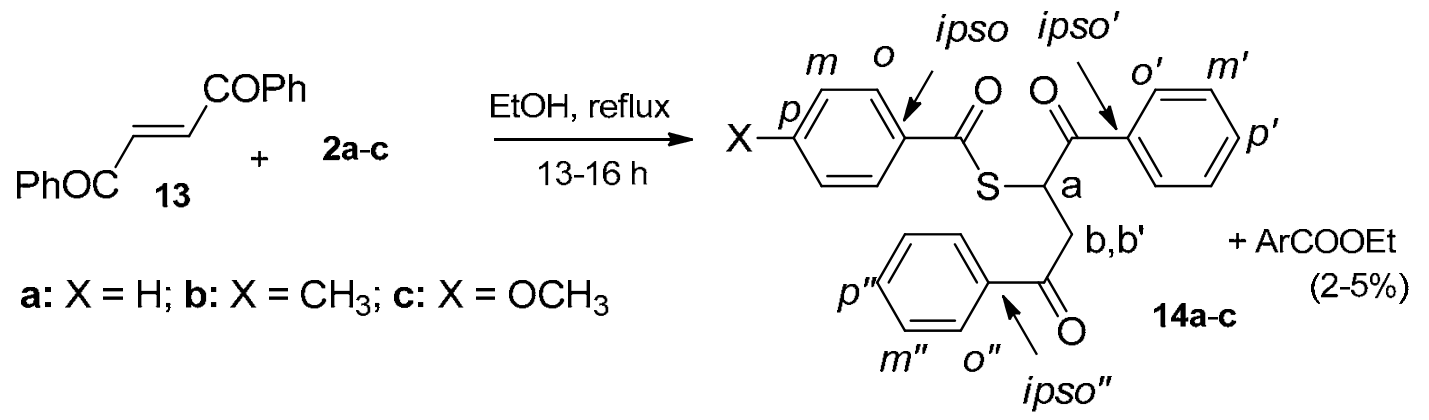

Scheme 6. Reaction of 2a-c with (E)-1,4-diphenylbut-2-ene-1,4-dione 13. 14a: 16 h, 80\%; 14b: 15 h, 82\%; 14c: 13 h, 84\%.

Surprisingly, on reacting 2a-c with $(E)$-1,4-diphenylbut-2-ene-1,4-dione $\mathbf{1 3}$ in refluxing ethanol, the reaction afforded $S$-(1-benzoyl-3-oxo-3-phenylpropyl) benzene-carbothioates 14a-c in good yields (Scheme 6). Again, mass spectrometry and microanalysis confirmed the molecular formulae, and IR and ${ }^{1} \mathrm{H} /{ }^{13} \mathrm{C}$ NMR supported the structures; connectivities were established using ${ }^{1} \mathrm{H}^{1} \mathrm{H}$ COSY, HMQC/HSQC, and HMBC studies. All HMQC and HSQC correlations were the same: HSQC was better-resolved as usual, ${ }^{14}$ and confirmed the correlations made less definitively with HMQC alone. All three products show nonequivalent phenyl ketone carbons in the ${ }^{13} \mathrm{C}$ NMR, requiring that these groups be on different carbons of the central two-carbon chain. H-b,b' are, of course, diastereotopic because of the chiral center at carbon a. The coupling constants between these two protons $(17.6-17.8 \mathrm{~Hz})$ are large for geminal coupling constants, but ${ }^{2} J$ values this large are precedented. ${ }^{13}$ The vicinal couplings of $10.0-10.2$ and $3.6-3.7 \mathrm{~Hz}$ are consistent with those for anti and gauche couplings observed in ethanes with three electronegative substituents, e.g., 1,1,2-trichloroethane. ${ }^{13}$

To shed light on the different selectivity, we reacted compounds 2a-c with 1,4-diphenylbut2-yne-1,4-dione 15. Again, mass spectrometry and microanalysis confirmed the molecular formulae, and IR and ${ }^{1} \mathrm{H} /{ }^{13} \mathrm{C}$ NMR supported the structures; connectivities were established using ${ }^{1} \mathrm{H}-{ }^{1} \mathrm{H}$ COSY, HMQC/HSQC, and HMBC studies. Each product of $\mathbf{1 6 a}$ is a mixture of two compounds (Scheme 7). Each compound contains two types of non-equivalent aroyl groups around an $s p^{3}$-hybridized core. There is one type of $s p^{3}$ carbon per compound, and ${ }^{1} \mathrm{H}$ integrals require there be one of each kind of benzoyl group per CHS. We assign the products as mixtures of the rac- and meso- twofold addition products 16a-c. 


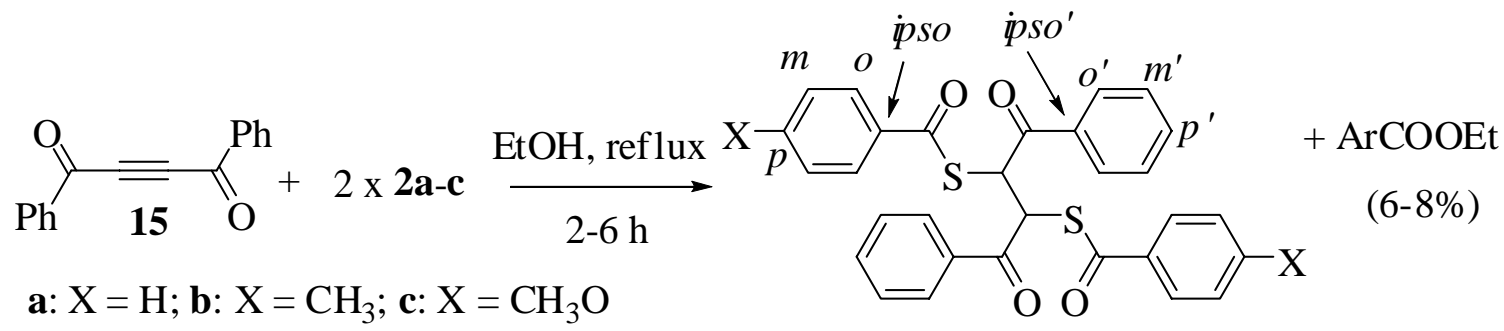

16a-c

Scheme 7. Reaction of 2a-c with 1,4-diphenylbut-2-yne-1,4-dione 15. 16a: 6 h, 75\%; 16b: 4 h, 77\%; 16c: 2 h, 80\%.

\section{Conclusions}

Diaroyl sulfides react with dimethyl acetylenedicarboxylate, ethyl propiolate, (E)-1,4diphenylbut-2-ene-1,4-dione and 1,4-diphenylbut-2-yne-1,4-dione to give products formally derived from conjugate addition of acylmercaptide to these $\pi$-acceptors.

\section{Acknowledgements}

We thank the National Science Foundation (CHE 03-42251) for the AV-400, which was purchased. We also thank the Assiut Microanalysis Center of Assiut University for carrying out the elemental analyses. Besides, we thank the Institute of Organic Chemistry, TU-Braunschweig. Germany for measuring the Mass spectroscopy.

\section{Experimental Section}

General. Melting points are uncorrected. ${ }^{1} \mathrm{H}$ NMR and ${ }^{13} \mathrm{C}$ NMR spectra were measured on Bruker AM-400 or AV-400 spectrometers (400.13 MHz for ${ }^{1} \mathrm{H}$ and $100.6 \mathrm{MHz}$ for ${ }^{13} \mathrm{C}$ ). Chemical shifts are in ppm, and coupling constants are in Hz. For preparative thin layer chromatography (PLC), glass plates $(20 \mathrm{x} 48 \mathrm{~cm})$ were covered with a slurry of silica gel Merck $\mathrm{PF}_{254}$ and air-dried using the solvents listed for development. Zones were detected by quenching of indicator fluorescence upon exposure to $254 \mathrm{~nm}$ UV light. Elemental analyses were carried in Assiut Microanalysis Center of Assiut University. Mass spectroscopy was performed with a Finnigan Mat 8430 spectrometer at $70 \mathrm{eV}$. IR spectra were run on a Shimadzu 470 spectrometer using $\mathrm{KBr}$ pellets; absorption frequencies $\left(v_{\max }\right)$ are reported in $\mathrm{cm}^{-1}$. 


\section{Starting materials}

The bis(dithiocarbamate) salt $\mathbf{1}$ was prepared according to reference 10 . Dimethyl acetylenedicarboxylate (or but-2-ynedioate), ethyl propiolate, (E)-1,4-diphenylbut-2-ene-1,4dione and 1,4-diphenylbut-2-yne-1,4-dione were bought from Fluka.

\section{General procedure of preparing diaroyl sulfides (or benzoic thioanhydrides) 2a-c}

To a $250 \mathrm{~mL}$ two-necked round-bottomed flask containing a solution of 1 (5 g, $12 \mathrm{mmol}$ ) dissolved in water $(200 \mathrm{~mL})$, polyethylene glycol $400(15 \mathrm{~g})$ was added. The reaction mixture was cooled to $0{ }^{\circ} \mathrm{C}$ and then a benzoyl chloride $(60 \mathrm{mmol})$ was added dropwise over 30 minutes. The reaction mixture was stirred at ambient temperature for 3-6 h (the reaction was followed by TLC analysis). The formed precipitate was filtered and then washed with cold ethanol (200 mL). The solid was then dried under vacuum, dissolved in dry dichloromethane $(50 \mathrm{~mL})$ and was subjected to column chromatography using dichloromethane. The obtained products 2a-c were recrystallized as illustrated in reference 1a. Compound 2a (2.6 g, 90\%), m.p. $=49{ }^{\circ} \mathrm{C}$ [lit. ${ }^{1 \mathrm{a}} 48-$ $49{ }^{\circ} \mathrm{C}$. Compound $2 \mathbf{b}\left(3.0\right.$ g, 94\%), m.p. $=89^{\circ} \mathrm{C}$ [lit. ${ }^{1 \mathrm{a}} 88-89{ }^{\circ} \mathrm{C}$. Compound $2 \mathbf{c}(3.5 \mathrm{~g}, 96 \%)$, m.p. $=95^{\circ} \mathrm{C}$ [lit. $\left.{ }^{1 \mathrm{a}} 94-95^{\circ} \mathrm{C}\right]$.

Reaction of 2a-c with dimethyl acetylenedicarboxylate 7. A mixture of 2a-c (1 mmol) and 7 (142 $\mathrm{mg}, 1 \mathrm{mmol}$ ) in absolute ethanol was heated under reflux for 4-5 h (the reaction was followed by TLC analysis). The solvent was then removed under vacuum and the residue was separated by preparative plate chromatography (silica gel, toluene: ethyl acetate 10:1). The obtained products 8a-c were isolated as the fastest zones, whilst the slowest zones contained ethyl benzoates (4-7\%) and were identified by comparison of $R_{f}$ values and IR spectra with authentic samples.

Dimethyl 2,3-bis(benzoylthio)succinate 8a. Colorless powder (0.25 g, 60\%), mp 134-6 ${ }^{\circ} \mathrm{C}$ (ethyl acetate). ${ }^{1} \mathrm{H}$ NMR $\left(\mathrm{CDCl}_{3}\right.$ ): 7.97 (d, $J=7.8,4 \mathrm{H} ; \mathrm{H}$-ortho), 7.60 (t, $J=7.4,2 \mathrm{H} ; \mathrm{H}-$ para), 7.46 ("t", $J=7.6,4 \mathrm{H} ; \mathrm{H}-\mathrm{meta}$ ), 5.32 (s, 2H; CHS), 3.79 (s, 6H; $\mathrm{CH}_{3}$ ). ${ }^{13} \mathrm{C} \mathrm{NMR}\left(\mathrm{CDCl}_{3}\right.$ ): 188.7 [C(=O)S], 170.1 [C(=O)O], 135.8 (C-ipso), 134.0 (C-p), 128.8 (C-m), 127.7 (C-o), $53.3\left(\mathrm{CH}_{3}\right)$, 46.6 (CHS). IR: 3090-3010 (w, Ar-CH), 2980-2820 (m, aliph-CH), 1740 (s, CO-ester), 1690 (s, $\mathrm{CO}), 1608(\mathrm{C}=\mathrm{C}), 1590$ (m, C=C). $\lambda_{\max }\left(\mathrm{CH}_{3} \mathrm{CN}, \lg \varepsilon, \mathrm{nm}\right): 360$ (3.4). MS (EI): $\mathrm{m} / \mathrm{z}(\%)=419$ [M+1] (54), 387 (9), 249 (6), 105 (100). $\mathrm{C}_{20} \mathrm{H}_{18} \mathrm{O}_{6} \mathrm{~S}_{2}$ (418.48): Calcd: C, 57.40; H, 4.34; S, 15.32. Found: C, 57.20, H, 4.34; S, 15.24.

Dimethyl 2,3-bis(4-methylbenzoylthio)succinate 8b. Colorless powder (0.29 g, 65\%), mp $162{ }^{\circ} \mathrm{C}$ (ethanol). ${ }^{1} \mathrm{H}$ NMR $\left(\mathrm{CDCl}_{3}\right.$ ): 7.86 (d, $J=8.0,4 \mathrm{H} ; \mathrm{H}-\mathrm{o}$ ), 7.24 (d, $J=8.1,4 \mathrm{H} ; \mathrm{H}-\mathrm{m}$ ), 5.30 (s, 2H; CHS), 3.78 (s, 6H; $\left.\mathrm{OCH}_{3}\right), 2.41$ (s, 6H; $\left.\mathrm{CCH}_{3}\right) .{ }^{13} \mathrm{C}$ NMR $\left(\mathrm{CDCl}_{3}\right): 188.2$ [C(=O)S], 170.2 [C(=O)O], 145.1 (C-p), 133.2 (C-ipso), 129.4 (C-o), 127.8 (C-m), $53.3\left(\mathrm{OCH}_{3}\right), 46.5$ (CHS), 21.8 ( $\mathrm{CCH}_{3}$ ). IR: 3100-3050 (w, Ar-CH), 2980-2870 (w, aliph.-CH), 1742 (s, CO-ester), 1682 (s, CO), 1612 (m, C=C), 1590 (s, C=C). $\lambda_{\max }\left(\mathrm{CH}_{3} \mathrm{CN}\right.$, lg $\varepsilon$, nm): 370 (3.5). MS (EI): m/z $(\%)=447\left[\mathrm{M}^{+}\right]$(53), 415 (10), 263 (6), 119 (100), 91 (10). $\mathrm{C}_{22} \mathrm{H}_{22} \mathrm{O}_{6} \mathrm{~S}_{2}$ (446.54): Calcd: C, 59.17; H, 4.97; S, 14.36. Found: 59.24; H, 5.00; S, 14.30. 
Dimethyl 2,3-bis(4-methoxybenzoylthio)succinate 8c. Colorless powder (0.34 g, 70\%), mp $182{ }^{\circ} \mathrm{C}$ (methanol). ${ }^{1} \mathrm{H}$ NMR (DMSO-d 6 ): 8.20 (d, $J=8.1$, 4H; Ar-H), 7.10 (d, $J=8.0$, 4H; Ar$\mathrm{H}), 5.26$ (br s, 2H; CHS), 3.90 (s, 6H; $\mathrm{ArOCH}_{3}$ ), 3.80 (s, 6H; $\mathrm{CO}_{2} \mathrm{CH}_{3}$ ). ${ }^{13} \mathrm{C}$ NMR (DMSO-d 6 ): $188.4[C(=\mathrm{O}) \mathrm{S}], 170.4$ [C(=O)O], 152.0 (C-para), 134.2 (C-ipso), 130.2 (C-ortho), 127.0 (Cmeta), $55.0\left(\mathrm{Ar}-\mathrm{OCH}_{3}\right), 53.8\left(\mathrm{CO}_{2} \mathrm{CH}_{3}\right), 46.6$ (CHS). IR: 3110-3090 (w, Ar-CH), 2990-2860 (m, aliph. CH), 1738 (s, CO-ester), 1685 (s, CO), 1625 (m, C=C). $\lambda_{\max }\left(\mathrm{CH}_{3} \mathrm{CN}, \lg \varepsilon\right.$, nm): 375 (3.8). MS (EI): $m / z(\%)=479$ [M $\mathrm{M}^{+}$(55), 447 (15), 279 (11), 135 (100), 107 (35), 76 (27). $\mathrm{C}_{22} \mathrm{H}_{22} \mathrm{O}_{8} \mathrm{~S}_{2}$ (478.54): Calcd: C, 55.22; H, 4.63; S, 13.40. Found: C, 55.00; H, 4.56; S, 13.30.

\section{Reaction of 2a-c with ethyl propiolate 11}

A mixture of compounds 2a-c (1 mmol) and 11 (0.098 g, $1 \mathrm{mmol})$ in absolute ethanol was heated under reflux for 5-7 h (the reaction was followed by TLC analysis). The solvent was then removed under vacuum and the residue was separated by preparative plate chromatography (silica gel, toluene: hexane 1:1). The obtained products 12a-c were recrystallized from the stated solvents. Ethyl benzoates were obtained as the slowest migrating zones in 2-4\% yields.

Ethyl (2Z)-3-(benzoylthio)acrylate 12a. Colorless powder $(0.17 \mathrm{~g}, 72 \%)$, mp $55-57{ }^{\circ} \mathrm{C}$ (ethanol). ${ }^{1} \mathrm{H}$ NMR $\left(\mathrm{CDCl}_{3}\right): 8.10$ (d, $J=10.2,1 \mathrm{H}$; H-b), 7.90-7.80 (m, 2H; Ar-H), 7.60-7.50 (m, 2H; Ar-H), 7.46-7.41 (m, 1H; Ar-H), 6.20 (d, $J=10.0,1 \mathrm{H} ; \mathrm{H}-\mathrm{a}), 4.20$ (q, $J=6.8,2 \mathrm{H} ; \mathrm{CH}_{2}$ ), 1.25 $\left(\mathrm{t}, J=6.8,3 \mathrm{H} ; \mathrm{CH}_{3}\right) .{ }^{13} \mathrm{C} \mathrm{NMR}\left(\mathrm{CDCl}_{3}\right): 186.4[C(=\mathrm{O}) \mathrm{S}], 166.4$ [C(=O)O], $137.0(C-\mathrm{b}), 131.2$ (C-o), 128.6 (C-m), 130.0 (C-p), 127.8 (C-ipso), $117.2(\mathrm{C}-\mathrm{a}), 60.0\left(\mathrm{CH}_{2}\right), 14.2\left(\mathrm{CH}_{3}\right)$. IR: 30863008 (w, Ar-CH), 2970-2870 (w, aliph-CH), 1735 (s, CO-ester), 1680 (s, CO), 1590 (m, C=C). $\lambda_{\max }\left(\mathrm{CH}_{3} \mathrm{CN}, \lg \varepsilon, \mathrm{nm}\right): 400$ (3.8). MS (EI): $\mathrm{m} / \mathrm{z}(\%)=236\left[\mathrm{M}^{+}\right]$(38), 191 (27), 131 (4), 105 (100), 77 (36). $\mathrm{C}_{12} \mathrm{H}_{12} \mathrm{O}_{3} \mathrm{~S}$ (236.29): Calcd: C, 61.00; H, 5.12; S, 13.57. Found: C, 60.87; H, 5.05; S, 13.45.

Ethyl (2Z)-3-(4-methylbenzoylthio)acrylate 12b. Colorless powder (0.19 g, 72\%), mp $70{ }^{\circ} \mathrm{C}$ (ethanol). ${ }^{1} \mathrm{H}$ NMR $\left(\mathrm{CDCl}_{3}\right): 8.08$ (d, $\left.J=10.3,1 \mathrm{H} ; \mathrm{H}-\mathrm{b}\right), 7.97$ (d, $J=8.2,2 \mathrm{H} ; \mathrm{H}-\mathrm{o}$ ), 7.30 (d, $J=$ 8.1, 2H; H-m), 6.19 (d, $J=10.3,1 \mathrm{H} ; \mathrm{H}-\mathrm{a}), 4.26$ (q, $J=7.1,2 \mathrm{H} ; \mathrm{CH}_{2} \mathrm{CH}_{3}$ ), 2.43 (s, 3H; $\mathrm{Ar}-\mathrm{CH}_{3}$ ), 1.33 (t, $\left.J=7.1,3 \mathrm{H} ; \mathrm{CH}_{2} \mathrm{CH}_{3}\right) .{ }^{13} \mathrm{C} \mathrm{NMR}\left(\mathrm{CDCl}_{3}\right): 187.0$ [C(=O)S], 166.3 [C(=O)O], 145.5 (Cp), 137.4 (C-b), 133.5 (C-ipso), 129.6 (C-m), 127.9 (C-o), 116.9 (C-a), $60.7\left(\mathrm{CH}_{2} \mathrm{CH}_{3}\right), 21.8$ (Ar$\mathrm{CH}_{3}$ ), $14.3\left(\mathrm{CH}_{2} \mathrm{CH}_{3}\right.$ ). IR: 3100-3050 (w, Ar-CH), 2950-2850 (w, aliph-CH), 1740 (s, CO-ester), 1680 (s, CO), 1600 (m, C=C). $\lambda_{\max }\left(\mathrm{CH}_{3} \mathrm{CN}, \lg \varepsilon, \mathrm{nm}\right): 400$ (3.8). MS (EI): $\mathrm{m} / \mathrm{z}(\%)=250\left[\mathrm{M}^{+}\right]$ (60), 205 (51), 131 (10), 119 (100), 91 (33), 77 (4), 65 (14). $\mathrm{C}_{13} \mathrm{H}_{14} \mathrm{O}_{3} \mathrm{~S}$ (250.31): Calcd: C, 62.38; H, 5.64; S, 12.81. Found: C, 62.20; H, 5.60; S, 12.76.

Ethyl (2Z)-3-(4-methoxybenzoylthio)acrylate 12c. Colorless powder $(0.22 \mathrm{~g}, 82 \%)$, mp $100{ }^{\circ} \mathrm{C}$ (ethanol). ${ }^{1} \mathrm{H}$ NMR $\left(\mathrm{CDCl}_{3}\right): 8.12$ (d, $J=10.0,1 \mathrm{H}$; H-b), 7.92 (d, $J=7.8,2 \mathrm{H}$; Ar-H), 6.90 (d, $J=$ 7.6, 2H; Ar-H), 6.20 (d, $J=10.0,1 \mathrm{H} ; \mathrm{H}-\mathrm{a}$ ), 4.22 (q, $J=6.8,2 \mathrm{H} ; \mathrm{CH}_{2}$ ), 3.95 (s, 3H; $\left.\mathrm{OCH}_{3}\right), 1.26$ $\left(\mathrm{t}, J=6.8,3 \mathrm{H}, \mathrm{CH}_{2} \mathrm{CH}_{3}\right) .{ }^{13} \mathrm{C} \mathrm{NMR}\left(\mathrm{CDCl}_{3}\right): 186.8$ [C(=O)S], 166.8 [C(=O)O], $150.0(\mathrm{C}-p)$, 137.2 (C-b), 134.2 (C-o), 130.2 (C-m), 126.2 (C-ipso), $117.0(\mathrm{C}-\mathrm{a}), 59.8\left(\mathrm{CH}_{2}\right), 50.8\left(\mathrm{OCH}_{3}\right)$, $14.3\left(\mathrm{CH}_{2} \mathrm{CH}_{3}\right)$. IR: 3086-3008 (w, Ar-CH), 1730 (s, CO-ester), 1690 (s, CO), 1590 (m, C=C). $\lambda_{\max }\left(\mathrm{CH}_{3} \mathrm{CN}, \lg \varepsilon, \mathrm{nm}\right): 400$ (3.8). MS (EI): $m / z(\%)=266\left[\mathrm{M}^{+}\right]$(65), 221 (54), 135 (100), 131 
(15), 107 (35), 76(24). $\mathrm{C}_{13} \mathrm{H}_{14} \mathrm{O}_{4} \mathrm{~S}$ (266.31): Calcd: C, 58.63; H, 5.30; S, 12.04. Found: C, 58.56; H, 5.20; S, 12.00 .

\section{Reaction of 2a-c with (E)-1,4-diphenylbut-2-ene-1,4-dione 13}

A mixture of compounds 2a-c (1 mmol) and $13(0.236 \mathrm{~g}, 1 \mathrm{mmol})$ in absolute ethanol was heated under reflux for 6-8 $\mathrm{h}$ (the reaction was followed by TLC analysis). The solvent was then removed under vacuum and the residue was separated by preparative plates chromatography (silica gel, toluene: ethyl acetate; 10:1)). The obtained products 14a-c which were obtained as the fastest migrating zones were recrystallized from the stated solvents. Ethyl aryloates were obtained as the slowest migrating zones in 2-5\% yields.

$S$-(1-Benzoyl-3-oxo-3-phenylpropyl) benzenecarbothioate 14a. Colorless powder (0.30 g, 80\%), mp 116-118 ${ }^{\circ} \mathrm{C}$ (ethanol). ${ }^{1} \mathrm{H}$ NMR $\left(\mathrm{CDCl}_{3}\right): 8.11$ (d, $\left.J=7.5,2 \mathrm{H} ; \mathrm{H}-o^{\prime}\right), 7.98$ (d, $J=7.5$, 2H; H-o”), 7.95 (d, $J=7.5,2 \mathrm{H} ; \mathrm{H}-\mathrm{o}$ ), 7.61-7.55 [m, 3H; H-p (all)], 7.50-7.43 [m, 6H; H-m (all)], 6.00 (dd, $J=10.1$, 3.6, 1H; H-a), 4.20 (dd, $J=17.8,10.1,1 \mathrm{H} ; \mathrm{H}-\mathrm{b}), 3.53$ (dd, $J=17.8,3.7,1 \mathrm{H}$; H-b’). ${ }^{13} \mathrm{C}$ NMR $\left(\mathrm{CDCl}_{3}\right)$ : 197.0 [(C=O)”], 195.9 [(C=O)'], $189.0(\mathrm{C}=\mathrm{O}), 136.0,135.1,134.1$ [Cipso (all)], 134.1, 133.6, 133.5 [C-p (all)], 128.9, 128.8, 128.7, 128.6 [C-m (all), C-o'], 128.2 (Co”), 127.6 (C-o), 41.9 (C-b), 41.2 (C-a). IR: 3100-3050 (w, Ar-CH), 2900 (w, aliph-CH), 1675 (s, CO), 1600 (m, C=C), 1450 (s, $\left.\mathrm{CH}_{2}\right) . \lambda_{\max }\left(\mathrm{CH}_{3} \mathrm{CN}, \lg \varepsilon, \mathrm{nm}\right): 340$ (3.2). MS (EI): $\mathrm{m} / \mathrm{z}(\%)=374$ [M $\mathrm{M}^{+}$(100), $356\left[\mathrm{M}^{+}-\mathrm{H}_{2} \mathrm{O}\right]$ (35), 252 (16), 236 (17), 208 (14), 105 (60), 77 (35). $\mathrm{C}_{23} \mathrm{H}_{18} \mathrm{O}_{3} \mathrm{~S}$ (374.45): Calcd: C, 73.77; H, 4.85; S, 8.56. Found: C, 73.60; H, 4.83; S, 8.45.

S-(1-Benzoyl-3-oxo-3-phenylpropyl) 4-methylbenzenecarbothioate 14b. Colorless powder (0.32 g, 82\%), mp $120^{\circ} \mathrm{C}$ (ethanol). ${ }^{1} \mathrm{H} \mathrm{NMR}\left(\mathrm{CDCl}_{3}\right)$ : 8.10 (dd, $J=7.3,1.3,2 \mathrm{H} ; \mathrm{H}-o$ '), 7.98 (dd, $J=7.2,1.3$, 2H; H-o”), 7.84 (d, $J=8.2,2 \mathrm{H}$; H-o), 7.57 (t, $J=7.4,2 \mathrm{H} ; \mathrm{H}-p^{\prime}, p^{\text {”) }), ~} 7.47$ (t, $J=7.7$, 2H; H-m'), 7.46 (t, $J=7.3,2 \mathrm{H} ; \mathrm{H}-m^{\prime}$ ), 7.24 (d, $J=8.2,2 \mathrm{H} ; \mathrm{H}-m$ ), 5.99 (dd, $J=10.2,3.7,1 \mathrm{H}$; H-a), 4.19 (dd, $J=17.8,10.2,1 \mathrm{H} ; \mathrm{H}-\mathrm{b}), 3.52$ (dd, $J=17.8,3.7,1 \mathrm{H} ; \mathrm{H}-\mathrm{b}$ ), 2.40 (s; 3H; $\mathrm{CH}_{3}$ ). ${ }^{13} \mathrm{C}$ NMR $\left(\mathrm{CDCl}_{3}\right)$ : 197.1 [(C=O)”], 196.1 [(C=O)'], $188.5(\mathrm{C}=\mathrm{O}), 145.2$ (C-p), 136.1, 135.1 (Cipso', ipso”), 133.5, 133.4 (C-p’,p”), 133.4 (C-ipso), 129.5 (C-m), 128.9, 128.7, 128.6 (Cm',m”,o'), 128.2 (C-o”), 127.6 (C-o), 42.0 (C-b), 41.1 (C-a), $21.8\left(\mathrm{CH}_{3}\right)$. IR: 3100-3065 (w, Ar$\mathrm{CH}$ ), 2980-2860 (w, aliph-CH), 1670 (s, CO), 1605 (m, C=C), 1455 (s, $\left.\mathrm{CH}_{2}\right) . \lambda_{\max }\left(\mathrm{CH}_{3} \mathrm{CN}, \lg \varepsilon\right.$,

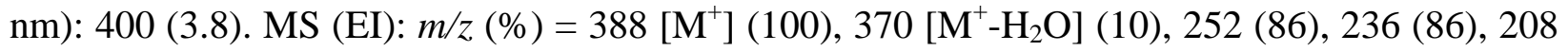
(34), 119 (60), 105 (60), 91 (31), 77 (42), 65 (10). $\mathrm{C}_{24} \mathrm{H}_{20} \mathrm{O}_{3} \mathrm{~S}$ (388.48): Calcd: C, 74.20; H, 5.19; S, 8.25. Found: C, 74.00; H, 5.12; S, 8.20.

S-(1-Benzoyl-3-oxo-3-phenylpropyl) 4-methoxybenzenecarbothioate 14c. Colorless powder (0.34 g, 84\%), mp $144{ }^{\circ} \mathrm{C}$ (acetone). ${ }^{1} \mathrm{H}$ NMR $\left(\mathrm{CDCl}_{3}\right): 8.12$ (dd, $J=7.3,1.3,2 \mathrm{H}$; H-o') 7.96 (dd, $J=7.2,1.3,2 \mathrm{H}$; H-o”), 7.76 (d, $J=8.2,2 \mathrm{H} ; \mathrm{H}-\mathrm{o}$ ), 7.60 (t, $J=7.4,2 \mathrm{H} ; \mathrm{H}-p, p$ '), 7.20-6.96 [m, 6H; H-m (all)], 5.98 (dd, $J=10.0,3.6,1 \mathrm{H} ; \mathrm{H}-\mathrm{a}$ ), 4.20 (dd, $J=17.6,10.0,1 \mathrm{H} ; \mathrm{H}-\mathrm{b}$ ), 3.94 (s, 3H; $\left.\mathrm{OCH}_{3}\right), 3.50$ (dd, $\left.J=17.8,3.6,1 \mathrm{H} ; \mathrm{H}-\mathrm{b}\right) .{ }^{13} \mathrm{C} \mathrm{NMR}\left(\mathrm{CDCl}_{3}\right): 197.0$ [(C=O)”], 196.0 [(C=O)'], $189.0(\mathrm{C}=\mathrm{O}), 160.9$ (C-p), 137.0, 136.2 (C-ipso', ipso”), 133.7, 133.2 (C-p',p”), 133.0, 129.2, 128.5, 128.0, 127.8 [C-m (all), o',o”] 127.0 (C-ipso), 116.0 (C-o), $55.0\left(\mathrm{OCH}_{3}\right), 42.2$ (Cb), 41.4 (C-a). IR: 3090-3000 (w, Ar-CH), 2950-2870 (w, aliph-CH), 1680 (s, CO), 1610 (m, $\mathrm{C}=\mathrm{C}), 1455$ (s, $\left.\mathrm{CH}_{2}\right) \cdot \lambda_{\max }\left(\mathrm{CH}_{3} \mathrm{CN}, \lg \varepsilon, \mathrm{nm}\right): 360$ (3.6). MS (EI): $\mathrm{m} / \mathrm{z}(\%)=404\left[\mathrm{M}^{+}\right](100)$, 
$386\left[\mathrm{M}^{+}-\mathrm{H}_{2} \mathrm{O}\right]$ (12), 252 (70), 236 (70), 208 (30), 135 (60), 107 (65), 76 (35). $\mathrm{C}_{24} \mathrm{H}_{20} \mathrm{O}_{4} \mathrm{~S}$ (404.48): Calcd: C, 71.27; H, 4.98; S, 7.93. Found: C, 71.10; H, 5.09; S, 8.08.

\section{Reaction of 2a-c with 1,4-diphenylbut-2-yne-1,4-dione 15}

A mixture of compounds 2a-c (1 mmol) and $\mathbf{1 5}(234 \mathrm{mg}, 1 \mathrm{mmol})$ in absolute ethanol was heated under reflux for 5-7 h (the reaction was followed by TLC analysis). The solvent was then removed under vacuum and the residue was separated by preparative plates chromatography (silica gel, toluene).

The obtained products 16a-c which were obtained as the fastest migrating zones were recrystallized from the stated solvents. Ethyl benzoates were obtained as the slowest migrating zones in 6-8\% yields.

S,S'-(1,4-Dioxo-1,4-diphenylbutane-2,3-diyl) dibenzenecarbothioate 16a. Colorless powder (0.38 g, 75\%), mp 174-176 ${ }^{\circ} \mathrm{C}$ (ethanol). IR: 3160-3080 (w, Ar-CH), 2980-2870 (w, aliph.-CH), 1700-1677 (s, CO), 1584 (m, C=C). $\lambda_{\max }\left(\mathrm{CH}_{3} \mathrm{CN}, \lg \varepsilon, \mathrm{nm}\right): 356,354$ (3.5,3.4). MS (EI): m/z $(\%)=510\left[\mathrm{M}^{+}\right]$(100), $373\left[\mathrm{M}^{+}-\mathrm{C}_{6} \mathrm{H}_{5} \mathrm{COS}\right]$ (40), 340 (24), 324 (30), 267 (34), 236 (35), 208 (26), 105 (34), 77 (60). $\mathrm{C}_{30} \mathrm{H}_{22} \mathrm{O}_{4} \mathrm{~S}_{2}$ (510.62): Calcd: C, 70.56; H, 4.34; S, 12.56. Found: C, 70.42; H, 4.28; S, 12.50. Major isomer: ${ }^{1} \mathrm{H}$ NMR $\left(\mathrm{CDCl}_{3}\right): 8.06$ (dd, $J=7.3,1.3,4 \mathrm{H} ; \mathrm{H}-o$ '), 7.88 (dd, $J=$ 7.3, 1.2, 4H; H-o), 7.59-7.50 (m, 4H; H-p,p'), 7.47-7.41 (m, 4H; H-m'), 7.36 (t, $J=7.8,4 \mathrm{H} ; \mathrm{H}-$ m), 6.31 (s, 2H; CHS). ${ }^{13} \mathrm{C}$ NMR $\left(\mathrm{CDCl}_{3}\right): 195.9$ [(C=O)'], 188.7 (C=O), 135.7 (C-ipso), 134.7 (C-ipso'), 134.1 (C-p), 133.8 (C-p'), 128.8-128.6 (C-o',m,m'), 127.7 (C-o), 47.7 (CHS). Minor isomer: ${ }^{1} \mathrm{H}$ NMR $\left(\mathrm{CDCl}_{3}\right)$ : 7.99 (dd, $J=7.3,1.3,4 \mathrm{H} ; \mathrm{H}-o$ '), 7.95 (dd, $J=7.3,1.2,4 \mathrm{H} ; \mathrm{H}-o$ ), 7.59-7.50 (m, 4H; H-p,p'), 7.47-7.41 (m, 8H; H-m,m'), 6.24 (s, 2H; CHS). ${ }^{13} \mathrm{C}$ NMR $\left(\mathrm{CDCl}_{3}\right)$ : 195.5 [(C=O)'], 189.3 (C=O), 135.8, 135.0 (C-ipso, ipso'), 134.7, 133.7 (C-p,p'), 128.8-128.6 (C-o',m,m'), 127.8 (C-o), 47.4 (CHS).

S,S'-(1,4-Dioxo-1,4-diphenylbutane-2,3-diyl) bis(4-methylbenzenecarbothioate) $16 \mathrm{~b}$. Colorless powder (0.42 g, 77\%), mp $192{ }^{\circ} \mathrm{C}$ (ethyl acetate). IR: 3160-3042 (w, Ar-CH), 29902860 (alip.-CH), 1700-1682 (s, CO), 1588 (m, C=C). $\lambda_{\max }\left(\mathrm{CH}_{3} \mathrm{CN}, \lg \varepsilon, \mathrm{nm}\right): 362,360$ (3.6,3.5). MS (EI): $m / z(\%)=538\left[\mathrm{M}^{+}\right]$(100), $387\left[\mathrm{M}^{+} 4-\mathrm{CH}_{3}-\mathrm{C}_{6} \mathrm{H}_{5} \mathrm{COS}\right]$ (50), 354 (26), 338 (34), 267 (36), 236 (18), 208 (20), 119 (38), 105 (50), 91 (40), 77 (28), 65 (24). $\mathrm{C}_{32} \mathrm{H}_{26} \mathrm{O}_{4} \mathrm{~S}_{2}$ (538.68): Calcd: C, 71.35; H, 4.86; S, 11.91. Found: C, 71.24; H, 4.82; S, 11.85. Major isomer: ${ }^{1} \mathrm{H}$ NMR ( $\mathrm{CDCl}_{3}$ ): 7.99 (d, $J=7.4,4 \mathrm{H} ; \mathrm{H}-o^{\prime}$ ), 7.84 (d, $J=8.2,4 \mathrm{H} ; \mathrm{H}-o$ ), 7.54 (t, $J=7.4,2 \mathrm{H} ; \mathrm{H}-p$ '), 7.43 (“t”, $J=7.6,4 \mathrm{H} ; \mathrm{H}-m$ '), 7.21 (d, $J=8.0,4 \mathrm{H} ; \mathrm{H}-m$ ), 6.22 (s, 2H; CHS), 2.39 (s, $\left.6 \mathrm{H} ; \mathrm{CH}_{3}\right) .{ }^{13} \mathrm{C}$ NMR (CDCl $)$ : 195.6 [(C=O)'], 188.7 (C=O), 145.1 (C-p), 135.1 (C-ipso'), 133.6 (C-p'), 133.2 (C-ipso), 129.4 (C-m), 128.67, 128.65 (C-o', m'), 127.9 (C-o), 47.3 (CHS), $21.8\left(\mathrm{CH}_{3}\right)$. Minor isomer: ${ }^{1} \mathrm{H}$ NMR $\left(\mathrm{CDCl}_{3}\right): 8.06(\mathrm{~d}, J=7.3,4 \mathrm{H} ; \mathrm{H}-\mathrm{o}$ ), $7.78(\mathrm{~d}, J=8.2,4 \mathrm{H} ; \mathrm{H}-\mathrm{o}), 7.54(\mathrm{t}, J=$ 7.4, 2H; H-p'), 7.44 (m, 4H; H-m'), 7.15 (d, J = 8.2, 4H; H-m), 6.22 (s, 2H; CHS), 2.39 (s, 6H; $\left.\mathrm{CH}_{3}\right) .{ }^{13} \mathrm{C}$ NMR $\left(\mathrm{CDCl}_{3}\right)$ : only the proton-bearing carbons can be observed directly. Nonprotonated carbons (italic) are inferred by analogy with major isomer. 195.6 [(C=O)'], 188.7 (C=O), 145.0 (C-p), 135.0 (C-ipso'), 133.8 (C-p'), 133.2 (C-ipso), 129.3 (C-m), 128.8, 128.7 (C$o$ ',m'), 127.8 (C-o), 47.7 (CHS), $21.7\left(\mathrm{CH}_{3}\right)$. 
S,S'-(1,4-Dioxo-1,4-diphenylbutane-2,3-diyl) bis(4-methoxybenzenecarbothioate) 16c. Colorless powder $(0.46 \mathrm{~g}, 80 \%), \mathrm{mp} 220^{\circ} \mathrm{C}$ (methanol). $\lambda_{\max }\left(\mathrm{CH}_{3} \mathrm{CN}, \lg \varepsilon, \mathrm{nm}\right): 390,386$ (3.7, 3.6). IR: 3090-3012 (w, Ar-CH), 2986-2850 (aliph.-CH), 1700-1680 (s, CO), 1590 (m, C=C), 1080 (C-O). MS (EI): m/z (\%) = 571 [M + 1] (30), 570 [M $]$ (100), 403 (45), 370 (35), 354 (38), 267 (60), 236 (37), 208 (20), 135 (70), 105 (48), 77 (28). $\mathrm{C}_{32} \mathrm{H}_{26} \mathrm{O}_{6} \mathrm{~S}_{2}$ (570.68): Calcd: C, 67.35; $\mathrm{H}, 4.59 ; \mathrm{S}, 11.24$. Found: C, 67.28; H, 4.46; S, 11.20. Major isomer: ${ }^{1} \mathrm{H}$ NMR $\left(\mathrm{CDCl}_{3}\right): 8.06$ (d, $J=7.4,4 \mathrm{H}$; H-o'), 7.84 (d, $J=8.2,4 \mathrm{H} ; \mathrm{H}-o$ ), 7.54 (t, $J=7.4,2 \mathrm{H} ; \mathrm{H}-p$ '), 7.43 (t, $J=7.6 \mathrm{~Hz}, 4 \mathrm{H}$;

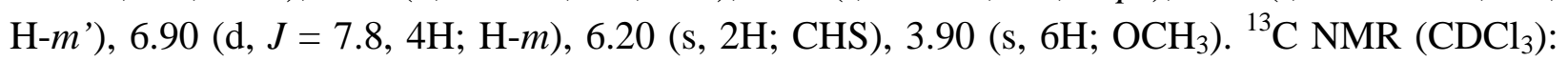
195.6 [(C=O)’], 188.7 (C=O), 160.8 (C-p), 138.0 (C-ipso), 133.4 (C-p), 129.2, 128.4, 128.0 (Cm,m',o'), 127.5 (C-ipso'), $115.7(\mathrm{C}-o), 54.8\left(\mathrm{OCH}_{3}\right), 48.3(\mathrm{CHS})$. Minor isomer: ${ }^{1} \mathrm{H}$ NMR $\left(\mathrm{CDCl}_{3}\right): 7.96$ (d, $\left.J=7.6,4 \mathrm{H} ; \mathrm{H}-o^{\prime}\right), 7.84$ (d, $\left.J=8.0,4 \mathrm{H} ; \mathrm{H}-\mathrm{o}\right), 7.54$ (t, $J=7.4,2 \mathrm{H} ; \mathrm{H}-p^{\prime}$ ), 7.43 (t, $J=7.8,4 \mathrm{H} ; \mathrm{H}-m$ '), 6.96 (d, $J=7.8,4 \mathrm{H} ; \mathrm{H}-m), 6.18$ (s, 2H; CHS), 3.95 (s, $\left.6 \mathrm{H} ; \mathrm{OCH}_{3}\right) .{ }^{13} \mathrm{C}$ NMR ( $\mathrm{CDCl}_{3}$ ): 195.4 [(C=O)’'], 188.4 (C=O), 160.4 (C-p), 138.2 (C-ipso), 133.0 (C-p), 129.4, 128.2, 127.8 (C-m,m’,o'), 127.3 (C-ipso'), 115.2 (C-o), $54.2\left(\mathrm{OCH}_{3}\right), 48.0(\mathrm{CH})$.

\section{References}

1. (a) Masumoto, H.; Tsutumi, H.; Kanda, T.; Komada, M.; Murai, T.; Kato, S. Sulfur Lett. 1989, 10, 103. (b) Koketsu, M.; Sekiya, Y.; Ishihara, H. Heteroatom Chem. 2003, 14, 633. (c) Deryagina, E. N.; Russavskaya, N. V.; Grabel'nykh, V. A. Russ. J. Gen. Chem. 2004, 74, 346.

2. (a) Shin, H. C.; Quinn, D. M. Lipids 1993, 28, 73. (b) Nedugov, A. N.; Pavlova, N. N. Zh. Org. Khim. 1992, 28, 1401. (c) Derbesy, G.; Harpp, D. N.; Rather, B.; Carroll, G. Sulfur Lett. 1992, 14, 199. (d) Harpp, D. N.; Gingras, M.; Aida, T.; Chan, T. H. Synthesis 1987, 1122. (e) Bonner, W. A. J. Am. Chem. Soc. 1950, 72, 4270.

3. Saucy, G.; Marbet, R.; Lindlar, H.; Isler, O. Helv. Chim. Acta 1959, 42, 1945.

4. Beyer, L.; Schöne, G. Tetrahedron Lett. 1970, 1959.

5. Mueller, W. H. J. Org. Chem. 1966, 31, 3075.

6. Gerecke, M.; Friedheim, E. A. H.; Brossi, A. Helv. Chim. Acta 1961, 44, 955.

7. Haugwitz, R. D. (E. R. Squibb \& Sons) U. S. Pat. 4338435, 1982; Chem. Abstr. 1982, 97, 182465p.

8. (a) Aly, A. A.; Hassan, A. A.; Ibrahim, Y. R. J. Chem. Res. 2008, 699. (b) Aly, A. A.; Hassan, A. A.; El-Sheref, E. M.; Mohamed, M. A.; Brown, A. B. J. Heterocycl. Chem. 2008, 45, 521. (c) Aly, A. A.; El-Shaieb, K. M. J. Chem. Res. 2007, 563. (d) Aly, A. A.; Ahmed, E. K.; El-Mokadam, K. M. J. Heterocycl. Chem. 2007, 44, 1431. (e) Aly, A. A.; Nour-El-Din, A. M.; Gomaa, M. A.-M.; Brown, A. B.; Fahmi, M. S. J. Chem. Res. 2007, 439. (f) Aly, A. A.; El-Shaieb, K. M. J. Chem. Res. 2007, 207. (g) Aly, A. A.; Hassan, A. A.; Gomaa, M. A.M.; El-Sheref, E. M. Arkivoc 2007, xiv, 1. (h) Aly, A. A.; Hassan, A. A.; El-Shaieb, K. M.; Shaker, R. M. Z. Naturforsch. 2005, 60b, 999. 
9. Aly, A. A.; Brown, A. B.; Abdel-Aziz, M.; Abuo-Rahma, G. E. A. A.; Radwan, M. F.; Ramadan, M.; Gamal-Eldeen, A. M. J. Hetercycl. Chem. 2009 (in press).

10. Guérin, D.; Carlier, R.; Lorcy, D. J. Org. Chem. 2000, 65, 6069.

11. Zhang, Y.-M.; Wei, T.-B.; Xian, L.; Gao, L.-M. Phosphorus Sulfur Silicon Relat. Elemts. 2004, 179, 2007.

12. Trofimov, B. A.; Nedolya, N. A.; Gerasimova, V. V.; Voronkov, M. G. (a) Zh. Org. Khim. 1988, 24, 2003. (b) Sulfur Lett. 1988, 8, 73.

13. Bovey, F. A. Nuclear Magnetic Resonance Spectroscopy, $2^{\text {nd }}$ ed.; Academic: San Diego, 1988; pp 612-614.

14. Simpson, J. H. Organic Structure Determination Using 2-D NMR Spectroscopy; Academic: Amsterdam, 2008; pp 124-132 (see especially p 131). 